\title{
COVID-19: Suggestion of Management Algorithm for Dentistry
}

\author{
Yunus Balel ${ }^{1 *}$, Mehmet Kemal Tumer ${ }^{1}$, Serkan Yildiz ${ }^{2}$ and Reza Tabrizi ${ }^{3}$ \\ ${ }^{1}$ Department of Oral and Maxillofacial Surgery, Faculty of Dentistry, Gaziosmanpasa University, Turkey \\ ${ }^{2}$ Department of Oral and Maxillofacial Surgery, Istanbul Topcular Oral and Dental Health Center, Turkey \\ ${ }^{3}$ Department of Oral and Maxillofacial Surgery, School of Dentistry, Shahid Beheshti University of Medical Sciences, Iran
}

*Corresponding author: Yunus Balel, Research Assistant, Department of Oral and Maxillofacial Surgery Faculty of Dentistry, Gaziosmanpasa University, Tokat, Turkey.
Received Date: July 23, 2020

Published Date: August 03, 2020

\begin{abstract}
Aim: COVID-19 disease, first seen in 2019, has spread to many countries and regions quickly, and the number of confirmed deaths and cases continues to increase. Effective infection control can prevent the spread of the virus which would lead to bringing the pandemic under control. As the SARS-CoV-2 virus is present in salivary secretions, the risk of cross-infection in dental health services is high. In this study, a review of and recommendations for preventive measures and treatment procedures that are needed in dentistry in consideration with published studies and guidelines are provide.
\end{abstract}

Materials and methods: Published studies and guidelines on COVID-19 were evaluated.

Results: A review of and recommendations for preventive measures and treatment procedures that are needed in dentistry in consideration with published studies and guidelines are provide.

Conclusion: During the pandemic, a simplified patient care flow chart was created in dentistry. The measures to be taken during patient care are summarized.

Keywords: CoVID-19; Dentistry; Prevention; Cross Infection; Patient Care

\section{Introduction}

Pandemics or pandemic diseases are epidemics that are widely spread such as a continent or around the world. Several epidemics were seen in the world from past to present such as Antonine Plague [1] (AD 165), Black Death [2] (1346-1353), and Third Cholera Pandemic [3] (1852-1860). COVID-19 disease, named by the World Health Organization (WHO) and first seen in the city of Wuhan in China at the end of December in 2019, is one of these epidemics [4-6].
The virus causing the disease was first named as 2019-nCoV [5]. Later, the name was revised as "severe acute respiratory syndrome coronavirus 2 (SARS-CoV-2)" by the Coronavirus Study Group of the International Virus Taxonomy Committee [7]. The World Health Organization (WHO) declared this global pneumonia epidemic as a public health emergency of international concern. As of June 10,2020 , the number of diagnosed cases is over 7 million, and the number of deaths is over 404.000. In this study, a review of and recommendations are provided for preventive measures and 
treatment procedures that are needed in dentistry in this pandemic that has a high risk of spread.

\section{General Symptoms and Modes of Transmission of the Disease}

In general, fever, coughing, expectorating, sore throat, and headache are the clinical symptoms seen in patients with new coronavirus pneumonia. The disease has the stages of mild disease, pneumonia, severe pneumonia, acute respiratory distress syndrome, sepsis and septic shock, respectively $[8,9]$.

Modes of transmission of the virus include inhalation of droplets formed during coughing or sneezing, and through oral and nasal mucosa. The entrance of the virus to the body is not limited to respiratory mucosa. The virus can enter through the conjunctiva of the eye as well $[10,11]$. In a study conducted by Sabino-Silva et al., three different paths were proposed for COVID-19 to appear in saliva [12]: First, COVID-19 in upper and lower respiratory tract enter the oral cavity through fluid droplets frequently exchanged by organs $[7,13,14]$. Second, COVID-19 present in blood can access the mouth through crevicular fluid which is an exudate unique to oral cavity including local proteins derived from proteins which are derivatives of extracellular matrix and serum [15]. Lastly, another way of COVID-19 to be present in the oral cavity is the minor and major salivary gland infection along with the release of particles in saliva through salivary tract. It should be noted that salivary gland epithelium cells can be infected with SARS-CoV-2 shortly after an infection in rhesus macaque which leads to the thought that salivary gland epithelium to be potentially an important source of the virus [16].

Virus can hang in the air for 3 hours through aerosols. It was found that the virus stays for 72 hours on stainless steel and plastic surfaces, for 8-24 hours on carton, and for 4-8 hours on copper surfaces [17]. Chest Computed Tomography and Real-Time Fluorescence polymerase chain reaction (RT-PCR) are used for diagnosis of the disease. Despite the high ratios of accuracy, these tests can't provide a $100 \%$ diagnosis of the disease $[18,19]$.

\section{Step 1- First Evaluation Prior To Physical Examination}

We recommend an evaluation of the general condition and the need for dental treatment through a form to be filled online prior to the physical examination of the patient. The contents of this form should include:

1) Did you have complaints of coughing, expectoration, shortness of breath, and change in taste and smell in the last 14 days?

2) Did you have fever in the last 14 days or if you had your temperature taken, what was your temperature?

3) Have you been in contact with someone who has been to other countries in the last 14 days? If yes, which country?
4) Have you been in crowded areas such as conferences, meetings, hospitals, etc. recently?

5) Are there at least two people who are confirmed for fever or respiratory problems, and have close contact with you in the last 14 days?

6) Do you have any systemic disease and are you in treatment for the disease?

7) What's the purpose of contacting your dentist?

8) If you have a dental x-ray taken in the last 6 months, please upload it to the system.

The first 5 questions of the form are geared towards an evaluation for COVID-19 pre-diagnosis. If one of the answers to those questions is "yes", the person should be recommended a 14day monitoring starting from the day of the last contact with people who are infected with SARS-CoV-2 or from the time they had been in high risk environments. They should seek medical help immediately after showing any symptoms including fever particularly, cough, shortness of breath or diarrhea [9]. The answer to the 6th question of the form is important for preventive measures in case of physical examination of the patient, and in need for consultation for the condition of the current systemic disease and adjusting the dosage of medications.

The last 2 questions are for the evaluation of the need for dental treatment. The X-ray taken in the last 6 months is evaluated to make a guess on the cause of the complaint the person described in the previous question. If one of the procedures requiring an emergency dental treatment described in step 2, the patient should be called in to the clinic for physical examination. The need of an emergency dental treatment of patients who responded "yes" to one of the first 5 questions should be evaluated by the dentist. The examination can be done by taking necessary and additional measures in the clinic, or in the patient's house or the hospital in which the patient is quarantined.

\section{Step 2- Physical Examination}

The physical examination of patients should be performed in private rooms. Mobile dental treatment services to be provided in patients' homes can be offered to prevent patients from leaving their homes and being in contact with other people and patients who contacted the dentist. The chair and the surfaces that the patient had touched before and after the examination should be cleaned with $70 \%$ ethanol or $0.1 \%$ sodium hypochlorite. Studies have shown that SARS, MERS, and SARS-CoV-2 coronaviruses can be disinfected with these solutions $[20,21]$. The safety measures to be taken by health professionals are published by WHO:[22]

1) Protective particle masks such as N95 masks certified by the USA National Institute for Occupational Safety and Health (NIOSH), European Union (EU) standard FFP2 or an equivalent should be used $[23,24]$. When disposable particle breathing 
device is used, a leak tightness control should be performed. It should be noted that beard may prevent the breathing device from fitting tightly [24].

2) Eye protection should be used (googles or face shield).

3) Clean, non-sterile, long-sleeve clothing and gloves should be worn. If the gowns are not water-resistant, then healthcare workers should use water-proof gowns for procedures that are expected to produce high levels of fluids that may penetrate the gown.

4) The number of people in the room should be limited to the absolute minimum required for patient care and support.

After the health professional takes preventive measures and before the patient is examined, $1 \%$ hydrogen peroxide or $0.2 \%$ povidone iodine gargle should be done [25]. Intraoral X-ray examination is the most common radiographic method for diagnosis in dentistry. However, it can induce saliva secretion and cough [26]. Therefore, extra oral radiography such as panoramic radiography and conical beam computed tomography should be preferred over intraoral radiography such as periapical X-ray.

Conditions Requiring Emergency Dental Treatment According to American Dental Association (ADA) Guidelines [27].

Dental emergencies that potentially threaten life:

1) Uncontrolled bleeding

2) Cellulite, or intraoral or extraoral swelling that potentially endangers the patient's airway and spread bacterial infection of soft tissue.

3) Trauma involving facial bones that threatens the patient's airway.

Emergency dental care focuses on the management of situations requiring emergency intervention to reduce severe pain and/or reduce the risk of infection, and to reduce the load of hospital emergency rooms. These should be treated minimally invasive as much as possible.

1) Severe toothache due to pulp inflammation.

2) Pericoronitis or pain in the third molar.

3) Cracked tooth causing pain or soft tissue trauma.

4) Dental Avulsion/tooth luxation.

5) Alveolar osteitis, dry socket formation following surgical procedure.

6) Abscess or localized bacterial infection causing local pain and swelling.

7) Dental treatments of patients requiring dental consultation prior to critical medical procedures (organ transplants and oncological treatment planning)
8) Permanent crown/bridge cementation if the temporary restoration is lost, broken or causes gum irritation.

\section{Other emergency dental care:}

1) Extensive dental caries or defective restorations causing pain.

2) Suture removal.

3) Denture adjustment on radiation/ oncology patients.

4) Adjustments or repair of dentures when function is impeded.

5) Changing temporary fillings in endodontic access cavities in patients experiencing pain.

6) Cut or adjustments of an orthodontic braces or apparatus that perforate the oral mucosa or causes ulceration

In case of need for emergency dental treatments, step 3 should be followed.

\section{Step 3-Dental Treatment Procedures}

If conditions requiring dental treatment emerge following the examination, safety measures described in step 2 should be continued. Patient should do a $1 \%$ hydrogen peroxide or $0.2 \%$ povidone iodine gargle again. Regardless of aerosol formation in the procedure, health professionals must wear goggles [28]. Bacteria spread to several surfaces in a dental clinic was shown as a result of aerosols produced from rotation devices used in dentistry [29-32]. In a study conducted by Al-Amad et al., it was shown that the use of rubber-dam in dental procedures, despite its clinical benefits, is associated with higher bacterial aerosol levels in the head area [22]. Thus, we do not recommend using rubber-dam in dental procedures during this period.

High speed dental aerators without valves to prevent suction may aspirate and eject fluids and debris accumulated during dental treatment procedures. Microbes including bacteria and viruses can contaminate the air and water tubes in dental units more and potentially cause cross infection [25]. Therefore, aerators with valves for suction prevention should be used. High suction potential drain apparatus and saliva suction devices should be used to reduce aerosol secretion during treatment [33,34]. Portable treatment frames can be used to limit the spread of aerosol. In life threatening conditions of excessive bleeding, abscess blocking the airway and facial trauma, the patient should be redirected to a specialized doctor.

\section{Management of severe pain caused by acute pulpitis}

1) Use a slow hand device with electric motor under high volume aspiration to access the pulp chamber

2) Use one file, wash the channel with irrigation solution

3) Dry the channel only with paper point or cotton pellet triple syringe is not recommended 
4) Use a single use pre-mixed blocker (for example, ZnOEug tube) for closing or place cotton pellet on channel opening for drainage and place temporary filling materials on it.

5) Plan an analgesic and, if necessary, antibiotic treatment

\section{Pericoronitis or pain in the third molar}

1) Postpone removal of impacted third molar as much as possible

2) Irrigate the surface below the area with pericoronitis with approved drugs (single use syringe and single use blunt tip needle)

3) In cases of trauma on the third molar from above, use a slow handheld device with single use drill (electric, if possible), do not perform irrigation, and slightly grind the cusp tip that is disturbing under high volume aspiration

4) Provide oral hygiene recommendations to the patient Plan a treatment of analgesic, and antibiotic -if necessary.

\section{Fractured tooth, tooth avulsion or luxation causing pain or soft tissue trauma}

1) Choose the appropriate standard procedure among tooth fracture, avulsion and luxation treatment procedures

2) In cases of repairing fractured tooth or splinting the affected tooth, use cotton pellet to dry rather than air/water syringe that causes aerosol formation.

\section{Alveolar osteoitis, dry socket formation following surgical procedure}

1) 1)Perform irrigation gently with physiological saline solution on the affected area

2) Use local analgesic or closing materials (like Alvogyl)

3) Provide oral hygiene recommendations to the patient. Plan an analgesic treatment

\section{Extensive dental caries or defective restorations causing pain}

1) "Chemomechanical Caries Removal" methods can be preferred to reduce aerosol formation to minimum

2) Manage the process with temporary restorative methods if possible (silver diamine fluoride, glass ionomers)

\section{Step 4-Medical Waste Management and Disinfection After Treatment}

Medical waste should be moved to the temporary storage area of the medical institution. Re-usable dental devices should be sterilized according to the "Infection Control: Device and Equipment Sterilizations Protocols" issued by the "Centers for Disease Control (CDC)" [35,36]. Areas within a diameter of 3 feet of the treatment area should be cleaned with $70 \%$ ethanol or
$0.1 \%$ sodium hypochlorite. The room should be aired with airconditioning systems with filters.

\section{Acknowledgement}

None.

\section{Disclosure of interest}

The authors declare that they have no competing interest.

\section{References}

1. Littman RJ, Littman ML (1973) Galen and the Antonine plague. Am J Philol 94: 243-255.

2. Spyrou MA, Tukhbatova RI, Feldman M, Joanna Drath, Sacha Kacki (2016) Historical Y. pestis Genomes Reveal the European Black Death as the Source of Ancient and Modern Plague Pandemics. Cell Host Microbe 19: 874-881.

3. Stine OC, Morris JGJ (2014) Circulation and transmission of clones of Vibrio cholerae during cholera outbreaks. Curr Top Microbiol Immunol 379: 181-193.

4. Zhu N, Zhang D, Wang W, Xingwang Li, Bo Yang (2020) A Novel Coronavirus from Patients with Pneumonia in China, 2019. N Engl J Med 382: $727-733$

5. Wu F, Zhao S, Yu B, Yan-Mei Chen, Wen Wang (2020) A new coronavirus associated with human respiratory disease in China. Nature 579: 265269.

6. Yang Y, Peng F, Wang R, Ming Yange, Kai Guan, Taijiao Jiang, et al. (2020) The deadly coronaviruses: The 2003 SARS pandemic and the 2020 novel coronavirus epidemic in China. J Autoimmun 109: 102434.

7. Coronaviridae Study Group of the International Committee on Taxonomy of Viruses (2020) The species Severe acute respiratory syndromerelated coronavirus: classifying $2019-\mathrm{nCoV}$ and naming it SARS-CoV-2. Nat Microbiol 5: 536-544.

8. Zhang X, Cai H, Hu J, Jiangshan Lian, Jueqing Gu, et al. (2020) Epidemiological, clinical characteristics of cases of SARS-CoV-2 infection with abnormal imaging findings. Int J Infect Dis 94: 81-87.

9. World Health Organization (2020) Home care patients with suspected Nov. coronavirus Infect. Present. with mild symptoms Manag. contacts.

10. To KK-W, Tsang OT-Y, Chik-Yan Yip C, Kwok-Hung Chan, Tak-Chiu Wu, et al. (2020) Consistent detection of 2019 novel coronavirus in saliva. Clin Infect Dis 71(15): 841-843.

11. Huang C, Wang Y, Li X, et al. (2020) Clinical features of patients infected with 2019 novel coronavirus in Wuhan, China. Lancet (London, England) 395: 497-506.

12. Sabino-Silva R, Jardim ACG, Siqueira WL (2020) Coronavirus COVID-19 impacts to dentistry and potential salivary diagnosis. Clin Oral Investig 24(4): 1619-1621

13. Li T, Lu H, Zhang W (2020) Clinical observation and management of COVID-19 patients. Emerg Microbes Infect 9: 687-690.

14. Wax RS, Christian MD (2020) Practical recommendations for critical care and anesthesiology teams caring for novel coronavirus (2019nCoV) patients. Can J Anaesth 67: 568-576.

15. Calisher C, Carroll D, Colwell R, et al. (2020) Statement in support of the scientists, public health professionals, and medical professionals of China combatting COVID-19. Lancet 395(10226): e42-e43.

16. Modern HealthCare (2020) Cancel. surgeries, COVID-19 patients could be "double whammy" Hosp.

17. Van Doremalen N, Bushmaker T, Morris DH, Myndi G Holbrook, Amandine Gamble, et al. (2020) Aerosol and Surface Stability of SARSCoV-2 as Compared with SARS-CoV-1. N Engl J Med 382(16): 1564-1567. 
18. Dai WC, Zhang HW, Yu J, Hua-Jian Xu, Huan Chen, et al. (2020) CT Imaging and Differential Diagnosis of COVID-19. Can Assoc Radiol J 71: 195-200.

19. Ai T, Yang Z, Hou H, Chenao Zhan, Chong Chen, et al. (2020) Correlation of Chest CT and RT-PCR Testing in Coronavirus Disease 2019 (COVID-19) in China: A Report of 1014 Cases. Radiology. 296(2): E32-E40.

20. Henwood AF (2020) Coronavirus disinfection in histopathology. J Histotechnol 43:102-104.

21. Kampf G, Todt D, Pfaender S (2020) Persistence of coronaviruses on inanimate surfaces and their inactivation with biocidal agents. J Hosp Infect 104: 246-251.

22. World Health Organization (2020) Infect. Prev. Control Dur. Heal. care when Nov. coronavirus ( $(\mathrm{n} \mathrm{CoV})$ "Infect. is suspected interim Guid.

23. World Health Organization (2020) Infect. Prev. Control epidemicpandemic-prone acute Respir. Dis. Heal. care.

24. World Health Organization (2008) How to perform a Part. Respir. seal check.

25. Peng X, Xu X, Li Y (2020) Transmission routes of 2019-nCoV and controls in dental practice. Int J Oral Sci 12: 1-9.

26. Vandenberghe B, Jacobs R, Bosmans H (2010) Modern dental imaging: a review of the current technology and clinical applications in dental practice. Eur Radiol 20: 2637-2655.

27. ADA (2020) Interim Guid. Minimizing Risk COVID-19 Transm.

28. Qing H, Li Z, Yang Z, Menghai Shi, Zixu Huang, et al. (2020) The possibility of COVID-19 transmission from eye to nose. Acta Ophthalmol 98(3): e388.
29. Shivakumar KM, Prashant GM, Madhu Shankari GS (2007) Assessment of atmospheric microbial contamination in a mobile dental unit. Indian J Dent Res Off Publ Indian Soc Dent Res 18: 177-180.

30. Rautemaa R, Nordberg A, Wuolijoki-Saaristo K, et al. (2006) Bacterial aerosols in dental practice-a potential hospital infection problem? ] Hosp Infect 64: 76-81.

31. Kedjarune U, Kukiattrakoon B, Yapong B (2000) Bacterial aerosols in the dental clinic: effect of time, position and type of treatment. Int Dent J 50: 103-107.

32. Al Maghlouth A, Al Yousef Y, Al Bagieh N (2004) Qualitative and quantitative analysis of bacterial aerosols. J Contemp Dent Pract 5: 91100.

33. Kohn WG, Collins AS, Cleveland JL, et al. (2003) Guidelines for infection control in dental health-care settings--2003. MMWR Recomm Rep 52: $1-61$.

34. ADA (2019) Am Dent Assoc Curr Policies Adopt. 1954-2019.

35. Centers for Disease Control and Prevention (2018) Dent. Handpieces Other Devices Attach. to Air Waterlines.

36. Centers for Disease Control and Prevention (2019) Statement Reprocess. Dent. Handpieces. 